\title{
Endocannabinoid-Hydrolysing Enzymes in the Post-Mortem Cerebellum of Humans Affected by Hereditary Autosomal Dominant Ataxias
}

\author{
Carmen Rodríguez-Cueto ${ }^{\mathrm{a}-\mathrm{c}}$ Cristina Benito ${ }^{\mathrm{a}, \mathrm{d}}$ Julián Romero $^{\mathrm{d}}$ \\ Mariluz Hernández-Gálvez ${ }^{\mathrm{a}-c, \mathrm{e}} \quad$ María Gómez-Ruiz ${ }^{\mathrm{a}-\mathrm{c}, \mathrm{e}} \quad$ Javier Fernández-Ruiz ${ }^{\mathrm{a}-\mathrm{c}}$ \\ ${ }^{a}$ Departamento de Bioquímica y Biología Molecular, Instituto Universitario de Investigación en Neuroquímica, \\ ${ }^{b}$ Centro de Investigación Biomédica en Red sobre Enfermedades Neurodegenerativas, and 'Instituto Ramón

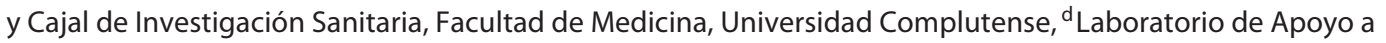 \\ la Investigación, Fundación Hospital Alcorcón, and eDepartamento de Psicobiología, Facultad de Psicología, \\ Universidad Complutense, Madrid, Spain
}

\section{Key Words \\ Cannabinoids · Endocannabinoid signalling system · Fatty acid amide hydrolase $\cdot$ Monoacyl glycerol lipase . \\ Cerebellum $\cdot$ Purkinje neurons $\cdot$ Spinocerebellar ataxias}

\begin{abstract}
Objectives: Spinocerebellar ataxias (SCAs) are characterized by a loss of balance and motor coordination due to degeneration of the cerebellum and its afferent and efferent connections. We recently found important changes in cannabinoid $C_{1}$ and $C B_{2}$ receptors in the post-mortem cerebellum of patients affected by different SCAs. Methods: We wanted to further explore this issue by analysing the two major endocannabinoid-hydrolysing enzymes, fatty acid amide hydrolase (FAAH) and monoacyl glycerol lipase (MAGL), in the post-mortem cerebellum of SCA patients and control subjects. Results: Immunoreactivity for the FAAH and MAGL enzymes was found in the granular layer, in Purkinje cells, in neurons of the dentate nucleus and in areas of white matter in the cerebellum of patients at levels frequently notably higher than those in control subjects. Using double-labelling procedures, we found co-localization of FAAH and MAGL with calbindin, supporting the presence of these enzymes in
\end{abstract}

Purkinje neurons. Conclusions: Degradative endocannabinoid enzymes are significantly increased in the cerebellum of SCA patients, which would presumably lead to reduced endocannabinoid levels. The identification of these enzymes in Purkinje neurons suggests a relationship with the pathogenesis of SCAs and suggests that the endocannabinoid system could provide potential therapeutic targets for the treatment of disease progression in SCAs.

(C) 2014 S. Karger AG, Basel

\section{Introduction}

Autosomal dominant spinocerebellar ataxias (SCAs) are a group of inherited neurodegenerative disorders, in which the most prevalent cases belong to the family of polyglutaminopathies, that also include Huntington's disease and that are primarily caused by excessive CAG repeats leading to expansion of a polyglutamine tract in different recipient proteins (i.e. huntingtin in Huntington's disease, frequently ataxins but also other proteins in SCAs) [1]. Despite its ubiquitous distribution, the mutant protein frequently affects only specific structures within the central nervous system in these disorders, the cerebel-

\section{KARGER}

E-Mail karger@karger.com www.karger.com/pat (c) 2014 S. Karger AG, Base

1015-2008/14/0813-0149\$39.50/0
Javier Fernández-Ruiz

Department of Biochemistry and Molecular Biology

Faculty of Medicine, Complutense University

ES-28040 Madrid (Spain)

E-Mail jjfr@med.ucm.es 
lum being the key structure affected in SCAs, which explains the specificity of neurological symptoms, i.e. motor incoordination and ataxia [2]. The age of onset of clinical symptoms is typically between 30 and 50 years of age, although early onset in childhood, as well as cases in which the pathology initiates after 60 years, have been reported for specific SCA subtypes, frequently related to longer or shorter polyglutamine expansions [3]. As in other polyglutamine diseases, SCAs are characterized by protein misfolding, failed or incomplete proteolysis and deposition and formation of intracellular protein aggregates, which represent the key neuropathological hallmark of these disorders [4]. Although there is some controversy in relation to the role of these aggregates in neurotoxicity, they appear to play a key role in eliciting transcription dysregulation, mitochondrial failure, excitotoxicity, alterations in calcium homeostasis, oxidative stress and local inflammatory events, which ultimately are responsible for the cell death that occurs in specific subpopulations of cerebellar neurons [5].

SCAs have not been studied yet for the possibility that they may benefit from disease-modifying therapies based on cannabinoid compounds, despite the well-demonstrated neuroprotective properties of these compounds $[6,7]$. The only study relating cannabinoids and SCAs was published recently and consisted of an analysis of the post-mortem cerebellum of SCA patients [8]. The objective was to elucidate the changes that the development of cerebellar degeneration produces in specific elements of the endocannabinoid signalling system, so that targeting the affected elements may serve to develop a novel form of therapy aimed at arresting/delaying the progression of this cerebellar degeneration, following the same schedule that has been used for other neurodegenerative disorders $[6,7,9]$. That study proved the occurrence of a notable upregulation of $\mathrm{CB}_{1}$ and $\mathrm{CB}_{2}$ receptors in SCA patients compared to control subjects, including their overexpression in neuronal subpopulations in which they are not usually located or their expression is weak, i.e. Purkinje cells [8]. These receptors, in particular the $\mathrm{CB}_{2}$ receptors, were also found to be increased in glial elements, e.g. reactive microglia, macrophages and astrocytes, in SCA patients [8]. The marked changes found in the two major receptors for endocannabinoids support a possible beneficial activity in SCAs of those cannabinoids selectively targeting the $\mathrm{CB}_{1}$ (they are effective in reducing excitotoxicity $[6,7]$ ) or the $\mathrm{CB}_{2}$ receptor (they are active in glial activation and associated local inflammatory events $[6$, $10])$, which is in addition to other neuroprotective properties of cannabinoids, for example their activity against
Table 1. Major characteristics of patients and control subjects whose post-mortem samples were used in this study (obtained from the NBB)

\begin{tabular}{rllll}
\hline Subject No. & $\begin{array}{l}\text { Age, } \\
\text { years }\end{array}$ & Gender & Diagnosis & $\begin{array}{l}\text { Post-mortem } \\
\text { delay, min }\end{array}$ \\
\hline 1 & 56 & female & control & 325 \\
2 & 62 & female & control & 480 \\
3 & 66 & female & control & 465 \\
4 & 83 & male & control & 315 \\
5 & 88 & male & control & 355 \\
6 & 54 & female & SCA3 & 315 \\
7 & 59 & female & SCA2 & 310 \\
8 & 61 & female & SCA & 325 \\
9 & 82 & male & SCA7 & 305 \\
10 & 86 & male & SCA6 & 395 \\
\hline
\end{tabular}

oxidative injury and their capability to act on several transcription factors (e.g. Nrf-2, NFkB) or to bind the peroxisome-proliferator activated receptors, all of which have already been demonstrated in numerous chronic neurodegenerative disorders $[6,9]$.

In our previous study [8], we placed emphasis on the two major endocannabinoid receptors, i.e. $\mathrm{CB}_{1}$ and $\mathrm{CB}_{2}$ receptors, but another two important endocannabinoid elements susceptible to pharmacological manipulation are the endocannabinoid-degrading enzymes fatty acid amide hydrolase (FAAH) and monoacyl glycerol lipase (MAGL). In this new study, both enzymes have been analysed, using immunohistochemical procedures, in the post-mortem cerebellum of SCA patients and control subjects.

\section{Materials and Methods}

\section{Subjects}

We used post-mortem human cerebella from control subjects and patients with a diagnosis of an autosomal dominant cerebellar ataxia that were obtained from the Netherlands Brain Bank (NBB), Institute for Neuroscience, Amsterdam, The Netherlands. All material was collected from donors for and from whom written informed consent for a brain autopsy and the use of material and clinical information for research purposes had been obtained by the NBB. Samples were received once fixed, paraffinized, sliced $(8 \mu \mathrm{m})$ and mounted on glass slides. Table 1 summarises the major characteristics (age, gender and post-mortem delay) of the individuals (patients and controls) included in our analyses. Unfortunately, the number of SCA types in the NBB, as well as in other biobanks that we contacted, is rather small, as SCAs are rare diseases, so that our experimental group had to be formed by only 1 subject for each of the most representative types of SCAs, particu- 
Table 2. List of antibodies used in immunohistochemical studies

\begin{tabular}{lllll}
\hline Antibody & $\begin{array}{l}\text { Dilution } \\
\text { for IHC }\end{array}$ & $\begin{array}{l}\text { Dilution } \\
\text { for IF }\end{array}$ & Class & Manufacturer \\
\hline FAAH & $1: 75$ & $1: 50$ & polyclonal & Santa Cruz Biotechnology, Santa Cruz, Calif., USA \\
MAGL & $1: 25$ & $1: 25$ & polyclonal & Abcam, Cambridge, UK \\
Calbindin D-28K & $1: 500$ & $1: 300$ & monoclonal & Sigma Chem., Madrid, Spain \\
Calbindin D-28K & & $1: 250$ & polyclonal & Millipore, Billerica, Mass., USA \\
\hline
\end{tabular}

IHC = Immunohistochemistry; IF = immunofluorescence.

larly those that are polyglutaminopathies. In any case, the neuropathological data provided by the biobank confirmed that all patients exhibited marked atrophy in the cerebellum and the pons (small size, presence of gliosis and evidence of neuronal loss) but with no important changes in the remaining brain, e.g. the substantia nigra was generally normal, the locus coeruleus was visible and the ventricular system was not dilated. We also performed a histopathological evaluation of all tissues received with the purpose to confirm the data provided by the biobank [8]. This evaluation was done prior to the use of these tissues for the immunohistochemical analysis of endocannabinoid receptors in the previous study [8] and of endocannabinoid-degrading enzymes in this study. We also considered the pharmacological information (medication and intoxication history) of SCA patients and control subjects provided by the NBB. Most SCA patients were treated with benzodiazepines (e.g. clonazepam, temazepam, diazepam), GABA-acting substances (e.g. baclofen, gabapentin) and, in a few cases, tricyclic antidepressants (e.g. amitriptyline). They were also sporadically treated with antibiotics (e.g. amoxicillin), and all of them received morphine during the last days/hours before death. One patient (subject 7) was treated with nabilone 6 years before death, but the treatment was not effective. Five patients with a genetic diagnosis of the most prevalent SCAs had to be dropped from the initial patient population $(n=10)$ because of a previous history of tobacco and/or alcohol addiction or because they consumed cannabis, so this is another important explanation for our small sample size. The medication and intoxication history of control subjects was also available and reviewed in the selection of cases to avoid any possible influence on the parameters examined in this study. Some of the control subjects had colon carcinoma and also received morphine during the last days/hours before death.

\section{Histochemical Techniques}

Immunohistochemistry

The protocol was used as described previously [11, 12] with slight modifications. Tissue sections were deparaffinised and washed extensively in $50 \mathrm{~mm}$ potassium phosphate-buffered saline (KPBS; pH 8). To obtain more efficient immunostaining, samples were subjected to an antigen retrieval procedure [13]. Briefly, tissue sections were placed into a stainless-steel pressure cooker containing Antigen Retrieval Solution, pH 9 (Dako Cytomation, Glostrup, Denmark). After heating under pressure for $2 \mathrm{~min}$, samples were removed and washed extensively in KPBS. Then, endogenous peroxidase was blocked by $30 \mathrm{~min}$ of incubation at room tempera- ture in peroxidase-blocking solution (Dako Cytomation). After several washes with KPBS, tissues were incubated with the primary antibody overnight at room temperature. Antibodies (below and table 2) were diluted in KPBS containing $1 \%$ bovine serum albumin (Sigma Chem., Madrid, Spain) and 1\% Triton X-100 (Sigma Chem.). After the incubation, sections were washed in KPBS, followed by incubation with biotinylated goat anti-rabbit antibody (1:200) or biotinylated horse anti-goat antibody (1:200) for $2 \mathrm{~h}$ at room temperature. Avidin-biotin complex (Vector Elite, Vector Laboratories, Burlingame, Calif., USA) and a DAB substrate-chromogen system (Dako Cytomation) were used to obtain a visible reaction product. Negative controls were used in each case to ensure antibody specificity. Sections were dehydrated, sealed and cover-slipped. A Leica DMRB microscope (Leica, Wetzlar, Germany) and a DFC300FX camera (Leica) were used for the observations and photography of the slides, respectively.

Quantification of FAAH and MAGL immunostaining was carried out on high-resolution digital microphotographs that were taken with $\mathrm{a} \times 20$ objective and under the same conditions of light and brightness/contrast. The microphotographs were used to measure the mean density of labelling in a selected area in the case of the granular layer and the white matter regions or the mean density of labelling in individual cells in the case of the Purkinje cells and the neurons of the dentate nucleus, using the analysis software Image (NIH, USA). Five images were taken for each case. We carried out a separate densitometrical analysis for each region. All data were expressed in arbitrary units.

\section{Immunofluorescence}

To identify specific cell populations, we performed co-localization studies with immunofluorescence combining the anti-FAAH or anti-MAGL antibodies with antibodies for specific markers for Purkinje cells (i.e. calbindin). After the antigen retrieval procedure, tissue sections were washed with Tris-buffered saline ( $\mathrm{pH}$ 7.5) before overnight incubation at room temperature with the anti-calbindin antibody followed by incubation with an Alexa 546 secondary antibody conjugate (Invitrogen, Carlsbad, Calif., USA) at $37^{\circ} \mathrm{C}$ for $2 \mathrm{~h}$, rendering red fluorescence. Afterwards, the primary antibodies for FAAH or MAGL were incubated overnight at room temperature after extensive washes in Tris-buffered saline, followed by incubation with Alexa 488 secondary antibody conjugate (Invitrogen), rendering green fluorescence. In the case of FAAH, the signal was visualized by incubation with biotinylated anti-goat secondary antibody, followed by streptavidin-Alexa 488 conjugate (Invitrogen). Sections were counter-stained with the nu- 


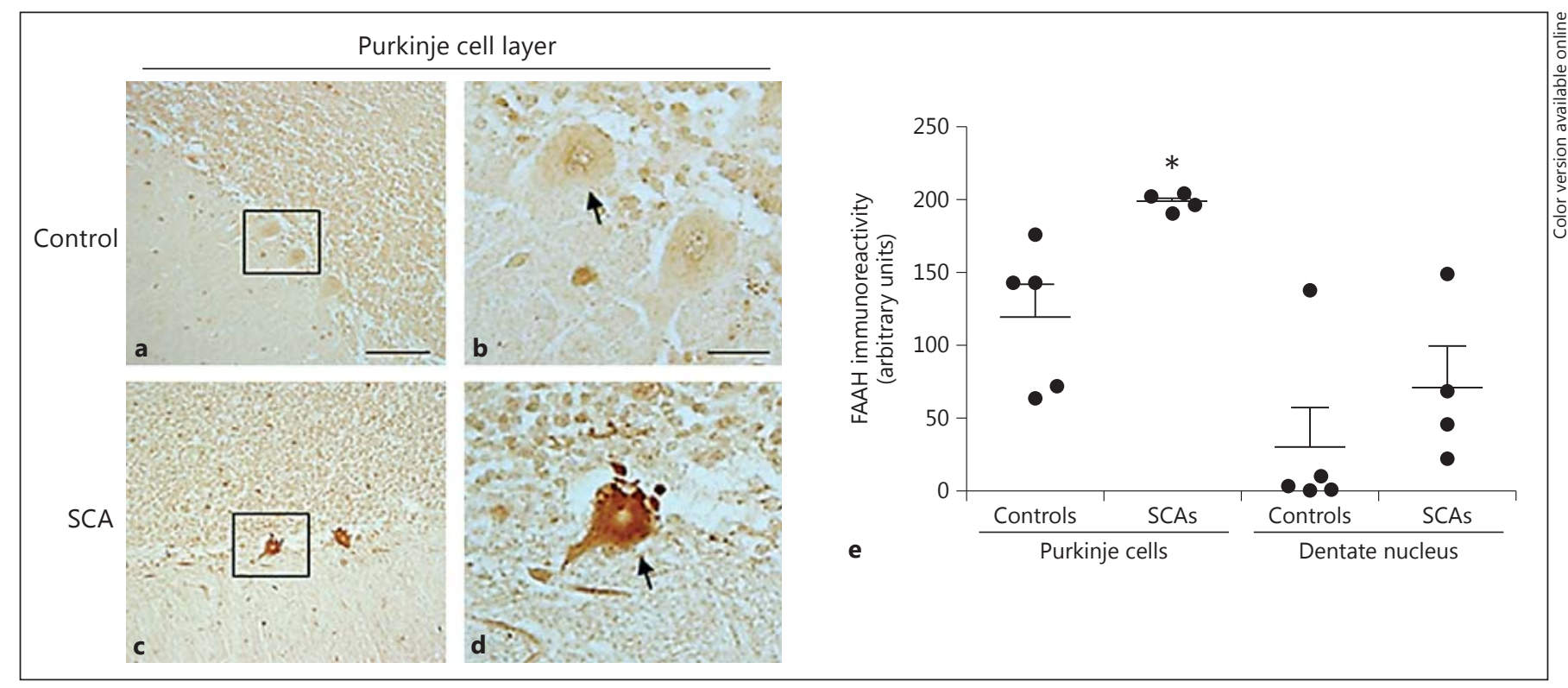

Fig. 1. a-d DAB immunostaining for the FAAH enzyme in the Purkinje layer of the cerebellum of SCA patients (c, d) and control subjects $(\mathbf{a}, \mathbf{b})$. The arrows indicate FAAH-positive cells. Scale bars $=100 \mu \mathrm{m}(\mathbf{a}, \mathbf{c})$ and $25 \mu \mathrm{m}(\mathbf{b}, \mathbf{d})$. The SCA microphotographs are from subject 9 (SCA7). e The scatter dot plot shows the quan-

clear stain TOPRO-3 iodide (Molecular Probes, Eugene, Oreg., USA) to visualize cell nuclei. To quench endogenous autofluorescence, tissue sections were treated with 1\% Sudan Black (Merck, Darmstadt, Germany) in $70 \%$ ethanol for 5 min and differentiated with $70 \%$ ethanol [14]. Sections were mounted onto glass slides with aqueous solution (Vectashield, Vector Laboratories).

Confocal Microscopy Analysis

Samples were viewed on a Leica TCS SP5 microscope system using argon and helium-neon lasers. Differential visualization of the fluorophores Alexa 488 (excitation $496 \mathrm{~nm}$ and emission 519 $\mathrm{nm}$ ), Alexa 546 (excitation $556 \mathrm{~nm}$ and emission $573 \mathrm{~nm}$ ) and TOPRO-3 (excitation $650 \mathrm{~nm}$ and emission $667 \mathrm{~nm}$ ) was accomplished through the use of specific filter combinations. Samples were scanned sequentially to avoid any potential for bleed-through of fluorophores.

Statistics

Data were assessed by Student's t test using the GraphPad software (version 5.0)

\section{Results}

Immunohistochemical Study of FAAH Enzyme in the Cerebellum of SCA Patients

We found positive immunostaining for FAAH, the enzyme responsible for endocannabinoid degradation, par- tification of FAAH immunostaining in Purkinje cells and the dentate nucleus of SCA patients and control subjects. Values correspond to the mean density of labelling in individual cells and are expressed as means \pm SEM of 5 subjects per group. Data were assessed by Student's t test. ${ }^{*} \mathrm{p}<0.05$. ticularly anandamide, in different parts of the cerebellum (fig. 1, 2). Our results showed a moderate increase in FAAH immunoreactivity in SCA patients compared to control subjects, but this increase reached statistical significance only in the Purkinje (fig. 1, FAAH-positive cells marked with arrows in $b$ and $d$ ) and granular (fig. 2, FAAH-positive cells marked with an arrow in d) layers, remaining only as a trend towards an increase in the white matter areas (fig. 2, FAAH-positive cells marked with arrows in $h$ and $l$ ) and showing no differences in the dentate nucleus (fig. 1).

FAAH immunoreactivity found in the Purkinje layer is apparently located in Purkinje neurons (arrow in fig. 1b), in particular in SCA patients (arrow in fig. 1d), in which the labelling was much more intense. In fact, the elevation of FAAH immunolabelling in Purkinje cells in SCA patients was found in the individual cells of those who survived degeneration (fig. 1). It is important to note that, given the marked neuronal loss found in the Purkinje layer and the dentate nucleus, the immunolabelling for the FAAH enzyme in SCA patients would be lower than that in controls if one considers the whole structure instead of the labelling in individual cells. This quantification also confirmed that the increase seen in individual cells occurred in all patients, even though they corre- 


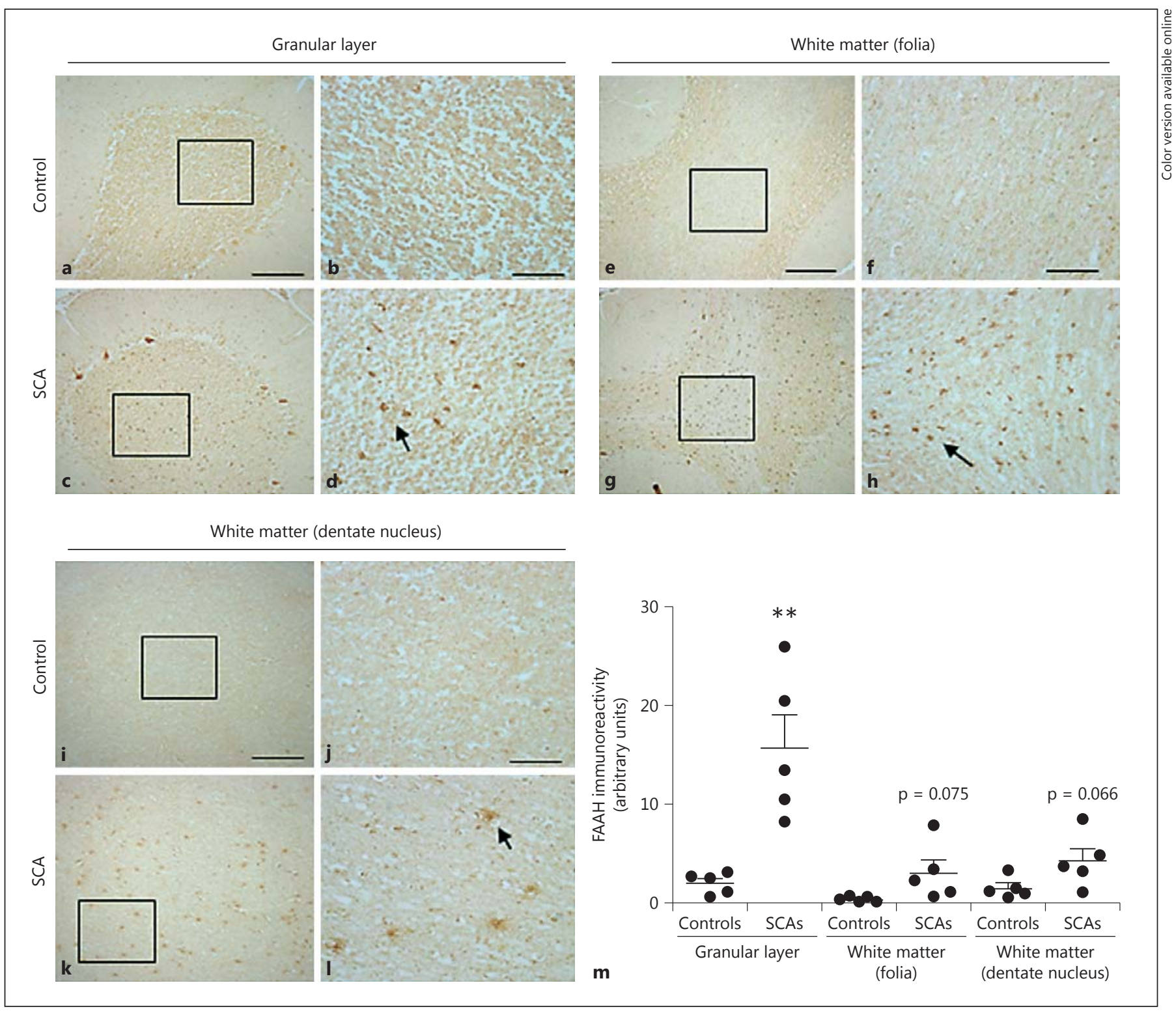

Fig. 2. a-I DAB immunostaining for the FAAH enzyme in the granular layer and the white matter areas of the cerebellum of SCA patients $(\mathbf{c}, \mathbf{d}, \mathbf{g}, \mathbf{h}, \mathbf{k}, \mathbf{I})$ and control subjects $(\mathbf{a}, \mathbf{b}, \mathbf{e}, \mathbf{f}, \mathbf{i}, \mathbf{j})$. The arrows indicate FAAH-positive cells. Scale bars $=200 \mu \mathrm{m}(\mathbf{a}, \mathbf{c}, \mathbf{e}, \mathbf{g}$, $\mathbf{i}, \mathbf{k})$ and $50 \mu \mathrm{m}(\mathbf{b}, \mathbf{d}, \mathbf{f}, \mathbf{h}, \mathbf{j}, \mathbf{I})$. The SCA microphotographs are from subject 9 (SCA7) for the granular layer and the white matter of the

sponded to different SCA types, and that the differences among the different SCA patients were not relevant (fig. 1). The presence of FAAH in Purkinje cells in SCA patients was further confirmed using double-labelling studies with antibodies against FAAH and calbindin (fig. 3). Co-localization was not so evident in controls as

folia and subject 10 (SCA6) for the white matter surrounding the dentate nucleus. $\mathbf{m}$ The scatter dot plot shows the quantification of FAAH immunostaining in the 3 structures in SCA patients and control subjects. Values correspond to the mean density of labelling in a selected area and are expressed as means \pm SEM of 5 subjects per group. Data were assessed by Student's t test. ${ }^{* *} \mathrm{p}<0.01$.

FAAH immunofluorescence was much weaker (fig. 3), although FAAH labelling was apparently identified in Purkinje cells using DAB immunostaining (fig. 1a, b), as has been previously reported $[12,15]$.

As mentioned above, FAAH immunoreactivity in the granular layer was significantly higher in SCA patients 


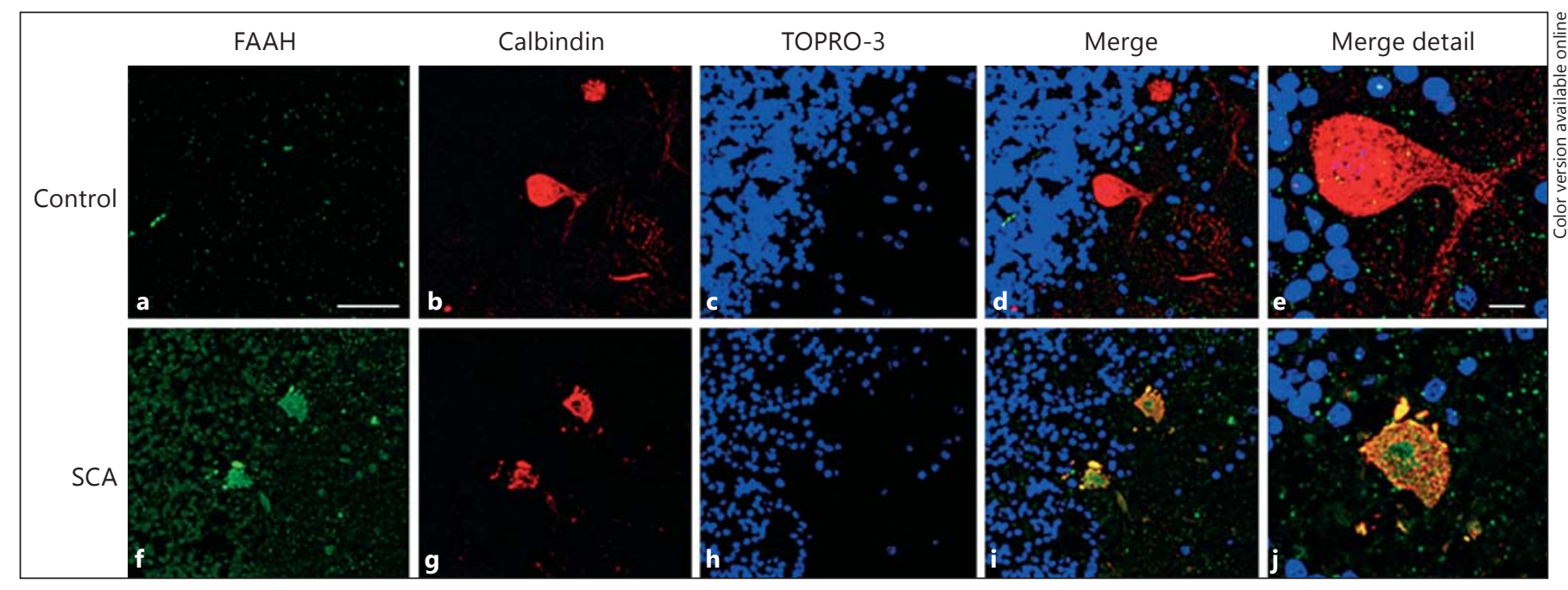

Fig. 3. Double-labelling immunofluorescence using antibodies for the FAAH enzyme and calbindin, as well as TOPRO-3 staining, in the Purkinje layer of the cerebellum of SCA patients $(\mathbf{f}-\mathbf{j})$ and control subjects (a-e), showing co-localization of the FAAH enzyme and calbindin in SCA patients but not in control subjects. Scale bars $=50 \mu \mathrm{m}(\mathbf{a}-\mathbf{d}, \mathbf{f}-\mathbf{i})$ and $10 \mu \mathrm{m}(\mathbf{e}, \mathbf{j})$. The SCA microphotographs are from subject 10 (SCA6). than in control subjects (fig. 2, FAAH-positive cells marked with an arrow in d), remaining only as a trend towards an increase in the white matter areas (fig. 2, FAAH-positive cells marked with arrows in $h$ and $l$ ). In these structures, the quantification was performed using a defined area, not the labelling in individual cells as in the case of Purkinje cells and neurons of the dentate nucleus. The quantification confirmed that, in the granular layer, the increase occurred in all patients, even though they corresponded to different SCA types, and that the differences among the different SCA patients were not relevant (fig. 2). The morphology of FAAH-containing cells in white matter structures seen with DAB immunostaining suggests that they could be astrocytes, as published elsewhere $[12,16]$.

\section{Immunohistochemical Study of MAGL Enzyme in the}

\section{Cerebellum of SCA Patients}

We also analysed MAGL, the enzyme responsible for 2-arachidonoyl glycerol degradation, in the cerebellum of SCA patients and controls (fig. 4-6). Using DAB immunostaining, MAGL was found to be located in Purkinje cells, as previously described [15], and in neurons of the dentate nucleus, in both cases at higher levels in SCA patients (fig. 4c, g, arrows in $\mathrm{d}$ and h) compared to control subjects (fig. 4a, e, arrows in b and f). Again this was seen in individual cells but not in the whole structure due to the neuronal loss evident in both areas. This quantifica- tion also confirmed that the increase occurred in all patients, even though they corresponded to different SCA types, and that the differences among the different SCA patients were not relevant (fig. 4). The location of MAGL in Purkinje cells was confirmed using double-labelling studies with this enzyme and calbindin, which revealed a high level of co-localization in SCA patients that was much less evident in control subjects (fig. 5). MAGL immunostaining was also evident in the Purkinje layer in cells other than the Purkinje neurons, possibly Bergmann glia, as previously described [17], and this was found in both SCA patients and control subjects (arrowheads in fig. 4b, d), although the labelling was much more evident in SCA patients (fig. 4d). This pattern was very similar to that of $\mathrm{CB}_{2}$ receptors [8], namely showing higher levels in SCA patients as they have more Bergmann gliosis. Lastly, as mentioned above, MAGL immunostaining found in neurons of the dentate nucleus was much more evident in SCA patients, with intense labelling distributed over the whole cell cytoplasm (fig. 4, arrow in h), whereas in controls, the MAGL signal was intense only in one small area located laterally in the cell cytoplasm (fig. 4, arrow in f). We assume that the labelling found in control subjects may represent an endogenous signal from lipofuscin rather than a specific signal for MAGL, although a similar response was not found when other neuronal markers (e.g. neuron-specific enolase) were used in the same control sections (data not shown). 
Immunoreactivity for MAGL was also evident in the granular layer (fig. 6). The quantification of this labelling revealed a relatively higher signal in SCA patients (fig. 6c, d) compared to controls (fig. 6a, b), although the differences did not reach statistical significance $(\mathrm{p}=0.185)$ due to a high variability in SCA patients, in particular in one of the cases (fig. 6). MAGL was also found in white matter areas, with similar levels in SCA patients and controls in the white matter surrounding the dentate nucleus (fig. 6i-1), but with significantly elevated levels in all SCA patients compared to controls in the case of the white matter of the folia (fig. 6e-h). Again it is important to remark that, in these structures, the quantification was performed using a defined area, not the labelling in individual cells as in the case of Purkinje cells and neurons of the dentate nucleus.

\section{Discussion}

The starting point for the present study was the data obtained in a previous one [8] that demonstrated the occurrence of a marked upregulation of $\mathrm{CB}_{1}$ and $\mathrm{CB}_{2}$ receptors in the post-mortem cerebellum of different SCA patients. In the previous study [8], we first performed an exhaustive analysis of several parameters to confirm the presence of histopathological signs of dysfunction/degeneration in the cerebellum of patients affected by different types of SCAs. As expected, we found that, compared to control subjects, the cerebellum of SCA patients presented (1) a notable loss of Purkinje cells and other cerebellar neuronal subpopulations (e.g. neurons located in the dentate nucleus), as well as strong evidence of a rare morphology in these cells (e.g. shrunken cell body, strongly reduced dendritic tree and axonal swellings) [8]; (2) a marked reactive microgliosis that was particularly evi-

Fig. 4. a-h DAB immunostaining for the MAGL enzyme in the Purkinje layer and dentate nucleus of the cerebellum of SCA patients $(\mathbf{c}, \mathbf{d}, \mathbf{g}, \mathbf{h})$ and control subjects $(\mathbf{a}, \mathbf{b}, \mathbf{e}, \mathbf{f})$. The arrows indicate the Purkinje cells that are MAGL positive, whereas the arrowheads indicate other MAGL-positive cells that presumably are Bergmann glial cells. Scale bars $=100 \mu \mathrm{m}(\mathbf{a}, \mathbf{c}, \mathbf{e}, \mathbf{g})$ and $25 \mu \mathrm{m}(\mathbf{b}$, $\mathbf{d}, \mathbf{f}, \mathbf{h}$ ). The SCA microphotographs are from subject 9 (SCA7) for the Purkinje layer and subject 10 (SCA6) for the dentate nucleus. i The scatter dot plot shows the quantification of MAGL immunostaining in the two structures in SCA patients and control subjects. Values correspond to the mean density of labelling in individual cells and are expressed as means \pm SEM of 5 subjects per group. Data were assessed by Student's t test. ${ }^{*} \mathrm{p}<0.05$; ${ }^{* * *} \mathrm{p}<$ 0.001 .

Endocannabinoid Enzymes in SCA Patients

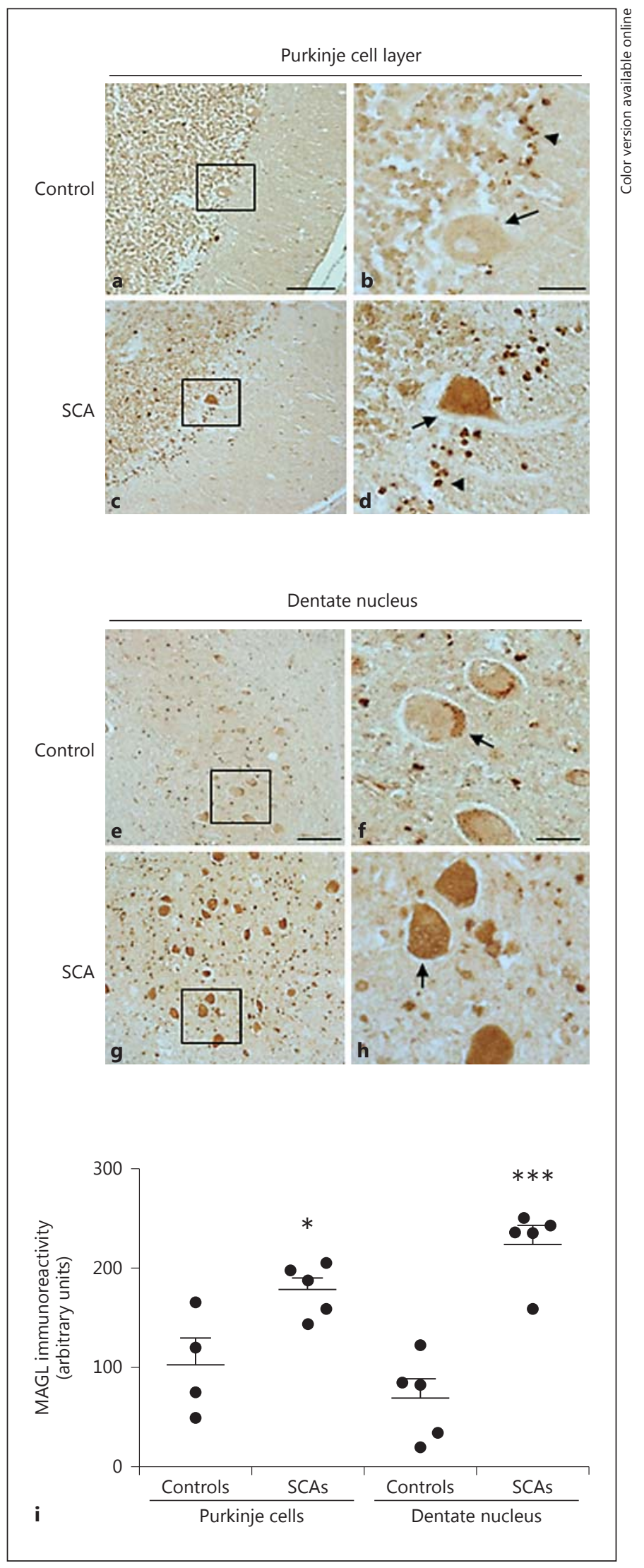

Pathobiology 2014;81:149-159 


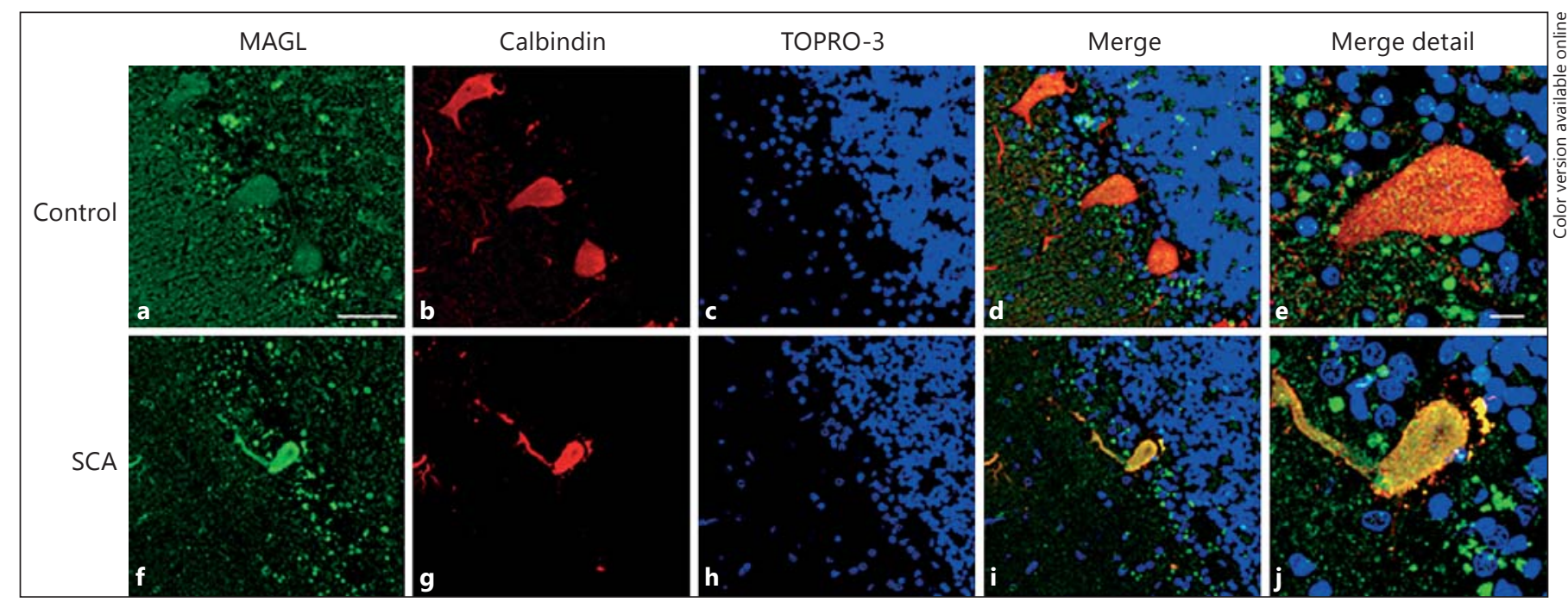

Fig. 5. Double-labelling immunofluorescence using antibodies for the MAGL enzyme and calbindin, as well as TOPRO-3 staining, in the Purkinje layer of the cerebellum of SCA patients $(\mathbf{f}-\mathbf{j})$ and control subjects (a-e), showing co-localization of the MAGL enzyme and calbindin, significantly in SCA patients but much more weakly in control subjects. Scale bars $=50 \mu \mathrm{m}(\mathbf{a}-\mathbf{d}, \mathbf{f}-\mathbf{i})$ and $10 \mu \mathrm{m}(\mathbf{e}$, j). The SCA microphotographs are from subject 8 (SCA).

are not identical in relation to the specific neuronal subpopulation that is most affected during the pathology. However, losses of Purkinje cells, as well as of other cerebellar neuronal subpopulations, have been documented in all SCA types included in this study [2]; moreover, the neuropathological information provided by the biobank and our preliminary examination of all post-mortem samples received allowed us to confirm these neuronal losses. In addition, despite the specific characteristics of each type of SCA, we found mostly the same responses for FAAH and MAGL enzymes as we already found in our previous study [8] for $\mathrm{CB}_{1}$ and $\mathrm{CB}_{2}$ receptors, and these responses were, in general, of a similar extent in all SCA types included in our study, which supports the validity of our experimental approach. Of course, it would have been better to concentrate on only one SCA type, but given that SCAs are rare diseases, it is difficult to collect a sufficient number of subjects of the same SCA type who are appropriately matched for gender, age and post-mortem delay or who do not have to be excluded due to potential influences on the results derived from their medication and intoxication histories. We were extremely careful in the selection of SCA cases and control subjects and, in fact, only $50 \%$ of the available cases could be included (see Materials and Methods). To this purpose, we excluded all cases of cannabis, alcohol or tobacco addiction and restricted the SCA group to cases of standard medication for this type of patient. Within the selected 


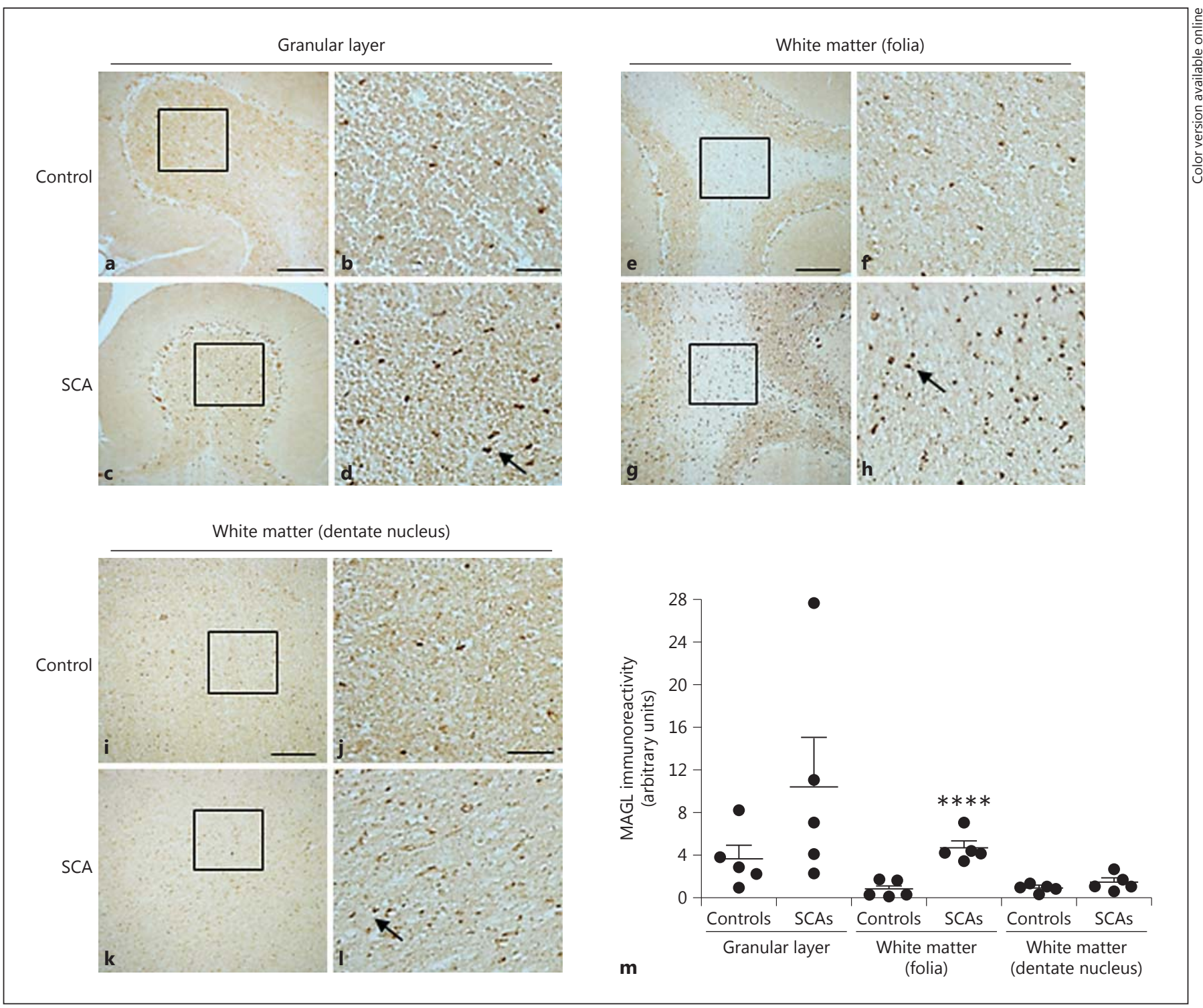

Fig. 6. DAB immunostaining for the MAGL enzyme in the granular layer and white matter areas of the cerebellum of SCA patients $(\mathbf{c}, \mathbf{d}, \mathbf{g}, \mathbf{h}, \mathbf{k}, \mathbf{I})$ and control subjects $(\mathbf{a}, \mathbf{b}, \mathbf{e}, \mathbf{f}, \mathbf{i}, \mathbf{j})$. The arrows indicate MAGL-positive cells. Scale bars $=200 \mu \mathrm{m}(\mathbf{a}, \mathbf{c}, \mathbf{e}, \mathbf{g}, \mathbf{i}, \mathbf{k})$ and $50 \mu \mathrm{m}(\mathbf{b}, \mathbf{d}, \mathbf{f}, \mathbf{h}, \mathbf{j}, \mathbf{I})$. The SCA microphotographs are from subject 9 (SCA7) for the granular layer and the white matter of the folia

and subject 6 (SCA3) for the white matter surrounding the dentate nucleus. $\mathbf{m}$ The scatter dot plot shows the quantification of MAGL immunostaining in the three structures in SCA patients and control subjects. Values correspond to the mean density of labelling in a selected area and are expressed as means \pm SEM of 5 subjects per group. Data were assessed by Student's t test. ${ }^{* * *} \mathrm{p}<0.005$.

cases, we found two potential problems, namely a patient who was treated with nabilone and the use of morphine in all SCA cases days or hours before death. However, we consider that these two factors had no influence on our results, as the nabilone treatment occurred 6 years before death in this patient, and the palliative treatment with morphine (or fentanyl) also occurred in those control

subjects who died of colon carcinoma, and the opioid did not produce any changes in the cerebellar levels of FAAH and MAGL in the present study, or of $\mathrm{CB}_{1}$ and $\mathrm{CB}_{2}$ receptors in our previous study [8].

Therefore, our data showed that the cerebellum of SCA patients responded with an increase in the endocannabinoid signalling mediated by both $\mathrm{CB}_{1}$ and $\mathrm{CB}_{2}$ 
receptors (data from our previous study [8]) but also with a presumed reduction in endocannabinoid levels due to enhanced endocannabinoid degradation by FAAH and MAGL enzymes, as shown in the present study. These two facts, which may a priori appear contradictory, may certainly be related to a first primary effect, i.e. the increase in $\mathrm{CB}_{1}$ and $\mathrm{CB}_{2}$ receptors, and a secondary and compensatory response increasing degradative enzymes with the purpose to reduce the availability of endocannabinoid ligands and recover normal endocannabinoid signalling. However, the opposite explanation is also possible. This would imply that the primary effect would be the increase in enzymes inactivating endocannabinoids, resulting in low levels of these endogenous ligands that would elicit a classical response in receptor-ligand regulation, i.e. upregulation of receptors and/or increase in their activity/signalling, aimed at compensating the reduced levels of endocannabinoids. In these post-mortem tissues, which correspond to cases of advanced stages of the disease, it is not possible to elucidate what the first effect and what the compensatory response is, so that we have recently initiated studies in mice transgenic for SCA3, the most prevalent SCA type, to investigate this question, for example using selective FAAH or MAGL inhibitors in experimental models of SCAs and determining their effects on disease progression. The analysis of the endocannabinoid levels in the cerebellum of these experimental models should also help in this regard.

On the other hand, the data obtained in both studies also suggest that the changes in these key elements of the endocannabinoid system in SCA patients may be instrumental, so that they may contribute somehow to the cerebellar dysfunction/degeneration during SCA pathogenesis. If this is the case, the pharmacological correction of these changes may have therapeutic benefits. This may be particularly relevant in the structures that contain the neuronal subpopulations most affected in SCAs, e.g. the Purkinje layer and dentate nucleus, in which the increase of FAAH and MAGL was seen in those cells that survived the degeneration, but, as the number of neurons in these structures decreases significantly in SCA patients, the labelling in the whole structure is rather reduced compared to control subjects. Alternatively, these changes may represent a protective response elicited to attenuate cerebellar dysfunction given the well-demonstrated neuroprotective function developed by this signalling system [6]. If this is the case, enhancing these responses may be the best option in the search of a cannabinoid-based therapy for SCAs. Therefore, an important consequence of our data is that the changes in the endocannabinoid signalling may be used to develop possible novel disease-modifying therapies based on the pharmacological activation/inhibition of those endocannabinoid elements altered in SCAs. These therapies may serve to control cytotoxic influences operating in SCA pathogenesis such as the phenomena of glial activation and local inflammatory events. Both questions have been explored in other similar neurodegenerative disorders $[6,9,21-25]$, and the issue has just reached the clinical scenario [7], so that these first data obtained in SCAs may be the start of a similar process in these cerebellar disorders.

In conclusion, the data collected in the present study, together with the results of our previous study [8], demonstrate that the endocannabinoid system is significantly altered in the cerebellum of SCA patients. These changes included an increase in $\mathrm{CB}_{1}$ and $\mathrm{CB}_{2}$ receptors [8] and the FAAH and MAGL enzymes, although these responses may be interpreted in different but complementary directions, as related somehow to SCA pathogenesis and/or as potentially serving for a novel pharmacological therapy. One important point related to these responses derives from the identification of the cellular substrates in which these responses occur; in particular, it is very important that these changes involve the Purkinje neurons, which are the main cells affected in SCAs and, according to our data, seem to contain both cannabinoid receptor types [8] and the two degradative enzymes. We are presently engaged in additional experiments aimed at confirming that the changes observed in the cerebellum of SCA patients can be reproduced in experimental models of SCAs (e.g. transgenic SCA3 mice) and to determine the timing of these alterations. We also plan to develop additional pharmacological experiments aimed at identifying the pharmacological profile of those cannabinoid compounds susceptible to the arrest/delay of disease progression in these transgenic mouse models.

\section{Acknowledgements}

This study was supported by the Ministerio de Ciencia e Innovación (SAF2009-11847 and SAF2010-16706), Centro de Investigación Biomédica en Red sobre Enfermedades Neurodegenerativas (CB06/05/0089) and Fundación Eugenio Rodríguez Pascual. Carmen Rodríguez-Cueto is a predoctoral fellow supported by the Formación de Personal Investigador Program-Ministry of Science, and Cristina Benito is a Juan de la Cierva postdoctoral fellow also supported by the Ministry of Science. The authors are indebted to Yolanda García-Movellán for her administrative support. 


\section{References}

$>1$ Klockgether T: Update on degenerative atax- 11 Benito C, Kim WK, Chavarría I, et al: A glial ias. Curr Opin Neurol 2011;24:339-345.

$>2$ Matilla-Dueñas A, Corral-Juan M, Volpini V, Sanchez I: The spinocerebellar ataxias: clinical aspects and molecular genetics. Adv Exp Med Biol 2012;724:351-374.

$>3$ Durr A: Autosomal dominant cerebellar ataxias: polyglutamine expansions and beyond. Lancet Neurol 2010;9:885-894.

-4 Orr HT: Cell biology of spinocerebellar ataxia. J Cell Biol 2012;197:167-177.

5 Fratkin JD, Vig PJ: Neuropathology of degenerative ataxias. Handb Clin Neurol 2012;103: 111-125.

-6 Fernández-Ruiz J, García C, Sagredo O, Gómez-Ruiz M, de Lago E: The endocannabinoid system as a target for the treatment of neuronal damage. Expert Opin Ther Targets 2010;14:387-404

$>7$ Fernández-Ruiz J, Moreno-Martet M, Rodríguez-Cueto $\mathrm{C}$, et al: Prospects for cannabinoid therapies in basal ganglia disorders. $\mathrm{Br} \mathrm{J}$ Pharmacol 2011;163:1365-1378.

8 Rodríguez-Cueto C, Benito C, FernándezRuiz J, Romero J, Hernández ML, GómezRuiz M: Changes in $\mathrm{Cb}_{1}$ and $\mathrm{Cb}_{2}$ receptors in the postmortem cerebellum of humans affected by spinocerebellar ataxias. Br J Pharmacol 2013, Epub ahead of print.

>9 Fernández-Ruiz J, Sagredo O, Pazos MR, et al: Cannabidiol for neurodegenerative disorders: important new clinical applications for this phytocannabinoid? Br J Clin Pharmacol 2013; 75:323-333.

-10 Fernández-Ruiz J, Romero J, Velasco G, Tolón RM, Ramos JA, Guzmán M: Cannabinoid CB2 receptor: a new target for controlling neural cell survival? Trends Pharmacol Sci 2007;28:39-45. endogenous cannabinoid system is upregulated in the brains of macaques with simian immunodeficiency virus-induced encephalitis. J Neurosci 2005;25:2530-2536.

12 Romero J, Hillard CJ, Calero M, Rábano A: Fatty acid amide hydrolase localization in the human central nervous system: an immunohistochemical study. Mol Brain Res 2002;100: 85-93.

13 Shi SR, Cote RJ, Taylor CR: Antigen retrieval techniques: current perspectives. J Histochem Cytochem 2001;49:931-937.

14 Schnell SA, Staines WA, Wessendorf MW: Reduction of lipofuscin-like autofluorescence in fluorescently labeled tissue. J Histochem Cytochem 1999;47:719-730.

15 Suárez J, Bermúdez-Silva FJ, Mackie K, et al: Immunohistochemical description of the endogenous cannabinoid system in the rat cerebellum and functionally related nuclei. J Comp Neurol 2008;509:400-421.

16 Benito C, Romero JP, Tolón RM, et al: Cannabinoid $\mathrm{CB} 1$ and $\mathrm{CB} 2$ receptors and fatty acid amide hydrolase are specific markers of plaque cell subtypes in human multiple sclerosis. J Neurosci 2007;27:2396-2402.

17 Tanimura A, Uchigashima M, Yamazaki M, et al: Synapse type-independent degradation of the endocannabinoid 2-arachidonoylglycerol after retrograde synaptic suppression. Proc Natl Acad Sci USA 2012;109:1219512200.
18 Ishikawa K, Mizusawa H, Fujita T, et al: Calbindin-D $28 \mathrm{k}$ immunoreactivity in the cerebellum of spinocerebellar degeneration. J Neurol Sci 1995;129:179-185.

19 Seidel K, Siswanto S, Brunt ER, den Dunnen W, Korf HW, Rüb U: Brain pathology of spinocerebellar ataxias. Acta Neuropathol 2012; 124:1-21.

20 Robitaille Y, Schut L, Kish SJ: Structural and immunocytochemical features of olivopontocerebellar atrophy caused by the spinocerebellar ataxia type 1 (SCA-1) mutation define a unique phenotype. Acta Neuropathol 1995 . 90:572-581.

21 Sagredo O, Pazos MR, Satta V, Ramos JA, Pertwee RG, Fernández-Ruiz J: Neuroprotective effects of phytocannabinoid-based medicines in experimental models of Huntington's disease. J Neurosci Res 2011;89:1509-1518.

22 Valdeolivas S, Satta V, Pertwee RG, Fernández-Ruiz J, Sagredo O: Sativex-like combination of phytocannabinoids is neuroprotective in malonate-lesioned rats, an inflammatory model of Huntington's disease: role of $\mathrm{CB} 1$ and $\mathrm{CB} 2$ receptors. ACS Chem Neurosci 2012;3:400-406.

23 Sagredo O, González S, Aroyo I, et al: Cannabinoid $\mathrm{CB} 2$ receptor agonists protect the striatum against malonate toxicity: relevance for Huntington's disease. Glia 2009;57:11541167.

24 Palazuelos J, Aguado T, Pazos MR, et al: Microglial CB2 cannabinoid receptors are neuroprotective in Huntington's disease excitotoxicity. Brain 2009;132:3152-3164.

-25 Benito C, Tolón RM, Pazos MR, Núñez E, Castillo AI, Romero J: Cannabinoid CB2 receptors in human brain inflammation. Br J Pharmacol 2008;153:277-285. 